\title{
Predicting the impact of climate change on the distribution pattern of Agamura persica (Dumeril, 1856) (Squamata: Gekkonidae) in Iran
}

\section{Seyyed Saeed Hosseinian Yousefkhani ${ }^{1}$, Mansour Aliabadian ${ }^{1,2, *}$, Eskandar Rastegar-Pouyani ${ }^{3}$ \& Jamshid Darvish ${ }^{1,2}$}

\author{
${ }^{1}$ Department of Biology, Faculty of Science, Ferdowsi University of Mashhad, Mashhad, Iran. \\ ${ }^{2}$ Research Department of Zoological Innovations (RDZI), Institute of Applied Zoology, Faculty of \\ Sciences, Ferdowsi University of Mashhad, Mashhad, Iran. \\ ${ }^{3}$ Department of Biology, Faculty of Science, Hakim Sabzevari University, Sabzevar, Iran. \\ *Corresponding author: aliabadi@um.ac.ir
}

\begin{abstract}
Species distribution modeling is an important tool that uses ecological data to aid in biological conservation. In the present study we used prediction methods, including maximum entropy (Maxent), to project the distribution of the Persian Spider gecko and the impact of climate change on its distribution in Iran. The results were consistent between models and indicated that two of the most important variables in determining distribution of Agamura persica are mean temperature of the wettest quarter and temperature seasonality. All of the models used in this study obtained high area-under-thecurve (AUC) values. Because of the nocturnal behavior of the species, these variables can directly affect species' activity by determining the vegetation type in habitat. Suitable habitats of Agamura persica were in two locations in eastern Iran and a third location in the central plateau. Habitat suitability for this species was increased in the last glacial maximum (LGM), at which time most parts of the Iranian Plateau were suitable (even southwest Iran). However, the suitable habitat area is restricted to the central part of the plateau in the current period. Predictions from four scenarios indicate that future habitat suitability will be patchy and that the central part of the plateau will remain the most important part of the species distribution.
\end{abstract}

KEYWORDS. Habitat suitability, Iran, nocturnal behavior, submountain region, vegetation type.

Hosseinian Yousefkhani S.S., Aliabadian M., Rastegar-Pouyani E. \& Darvish J. 2017. Predicting the impact of climate change on the distribution pattern of Agamura persica (Dumeril, 1856) (Squamata: Gekkonidae) in Iran. Belgian Journal of Zoology 147 (2): 137-142. https://doi.org/ 10.24696/bjz.2017.11

\section{Introduction}

Climate change is an important focus of current ecological and evolutionary study that opens new challenges in biodiversity conservation (SALA et al. 2000). During recent years, climate change may have been the main cause of species extinctions (POUNDS et al. 2006). Additionally, global warming has changed the distribution of species towards the poles and, in some cases, has caused local populations to shift to higher elevations (ROOT et al. 2003). These changes affect species and cause major problems for breeding (WALTHER et al. 2002). Predictive programs such as those used for species distribution modeling can help conservation biologists address these issues and develop procedures to protect species that are at risk. 
Reptiles are ectothermic vertebrates, and are distributed in most parts of the world except for the poles (VITT \& CALDWELl 2013). These animals are affected by the climate, and especially by temperature. Climate change from the last glacial period has been considerable and is predicted to increase because many scenarios have predicted high levels of greenhouse gases by 2050 and 2070 (CANADELL et al. 2007). Recent IPCC reports have provided four categories as different climate layers $(2.6,4.5,6.0$ and 8.5) and each category includes a particular scenario for future climate change in relation to greenhouse gas emissions.

In Iran, nocturnal lizards belonging to family Gekkonidae live in desert regions, including Agamura persica, which is distributed in central and eastern Iran and restricted to submountain regions with small rocks on the ground (ANDERSON 1999). The species' distribution range is only known from central (the most western part of Qom province), north eastern (Khorasan Razavi province), and eastern Iran (South Khorasan and Sistan and Baluchistan provinces). Additionally, its range extends eastward to Afghanistan (Kandahar) and Pakistan (Nushki and Kharan) (Fig. 1) (SzCZERBAK \& GolubeV 1996; ANDERSON 1999).

One important tool in the study of biogeography and ecology is the use of ecological niche models (ENM) to find the best fitted model to describe the actual distribution or habitat suitability of a species (FRANKLIN 2010). Attempts to predict the most appropriate regions for distribution of a given species have used Maximum Entropy (MaxEnt) models, which are based only on presence (OP) records (PHILliPS et al. 2006). We cannot predict species occurrence using only presence records, but can only predict habitat suitability. OP models do not have closed algorithms; they calculate the average values of grid cells and try to find the proportion of similar grids on the map based upon bioclimatic layers (KÉRY et al. 2010). Additionally, the OP depends on the cell size, and bias results in an incorrect model if the size is large or the number of records are few (CHAKRABORTY \& NEWTON 2011; KeIL et al. 2013).

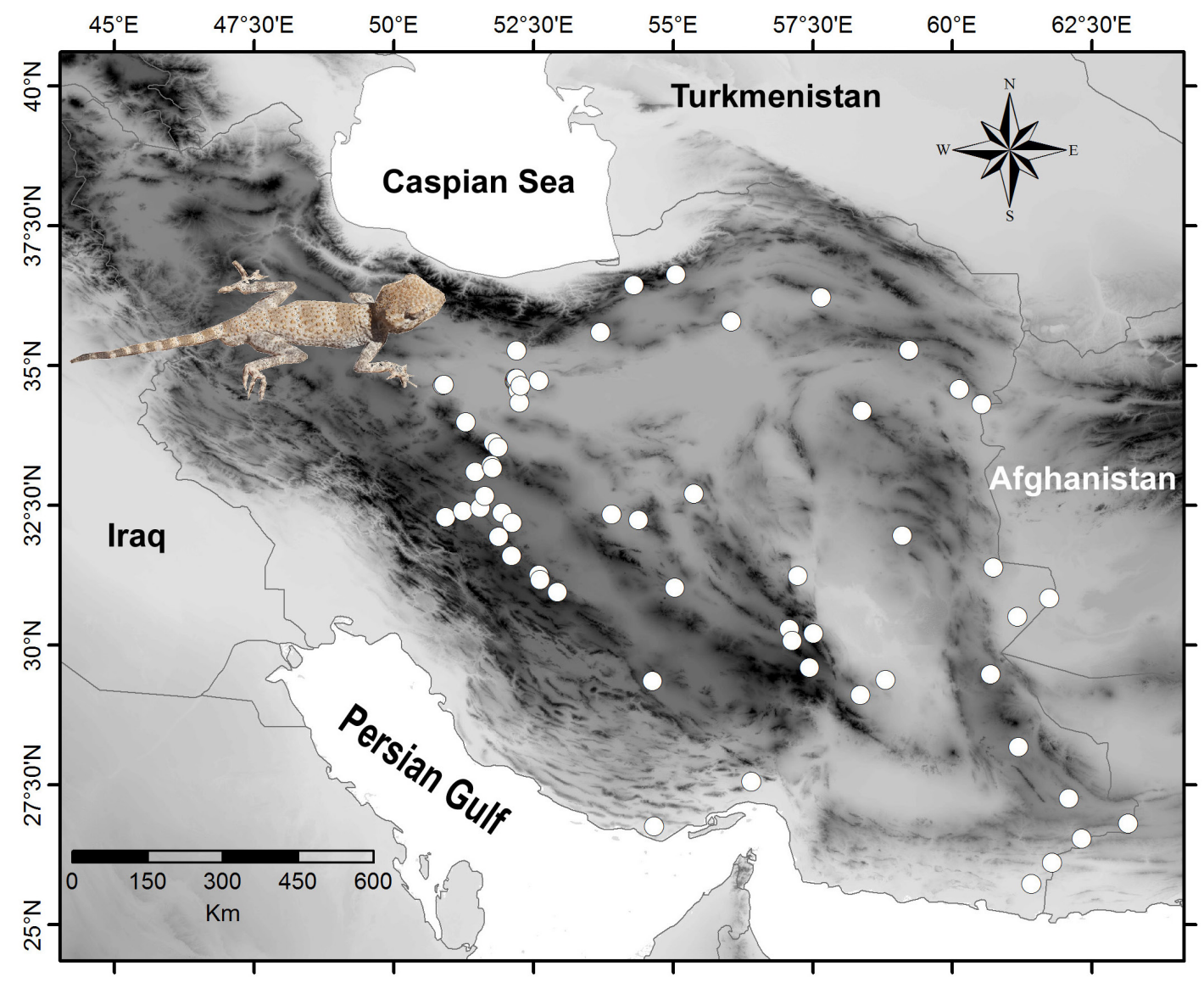

Figure 1 - Map of Iran showing distribution points of Agamura persica used in this study. 
In the present study, we modeled habitat suitability for Agamura persica for five time periods (Last Interglacial, Last Glacial Maximum, present, 2050 and 2070) using MaxEnt method. Additionally, we distinguished the important environmental variables and discussed their changes between time periods for the range of species distributions. The objectives of this study were to (1) predict the habitat suitability and the effect of climate change using MaxEnt, and (2) determine the main environmental variables influencing suitable habitat regions.

\section{Material and methods}

\section{Occurrence and environmental data}

Species occurrence records were obtained from three main sources: 1-our fieldwork within Iranian borders; 2- previously published literature; and 3- the GBIF website (www.gbif.org). Finally, 163 records (decimal degree) were gathered as presence records.

In total, 19 bioclimatic layers were downloaded as the present (1950-2010), the Last Inter Glacial (LIG) (120000 years ago) and Last Glacial Maximum (LGM) from www.worldclim.org and future layers under the recent IPCC reports were obtained for 2050 and 2070 as four scenarios $(2.6,4.5,6.0$, 8.5) from www.ccafs-climate.org. All layers were downloaded in 30 arc-second resolution and cropped for Iranian boundaries using ArcGIS 10.3. Environmental layers and records were examined using Openmodeller v 1.0.7 (DE SOUZA MUÑOZ et al. 2011) to find the highly correlated variables. The Pearson correlation method was used in SPSS 16.0 to find correlative variables greater than 0.75 . Variables with lower correlations than 0.75 were chosen to run the models. BIO4 (Temperature Seasonality); BIO6 (Minimum Temperature of Coldest Month); BIO8 (Mean Temperature of Wettest Quarter); BIO9 (Mean Temperature of Driest Quarter); BIO11 (Mean Temperature of Coldest Quarter) layers were chosen to run the models.

\section{Modeling of species distribution}

Species distributions were modelled using environmental variables belonging to different periods. We used MaxEnt 3.3.3e (PHILLIPS et al. 2006) to build the species distribution for the present and project it to the past and future . MaxEnt used only presence (OP) records for predicting suitable area (ELITH et al. 2011). Thirty percent of the data were used as test data and the rest were considered as training data. All setting parameters were used as default: maximum number of iterations $=500$; convergence threshold $=10^{-5}$; multiplier regularization $=1$ and ten replicates (PHILLIPS et al. 2006). The area under receiver-operating-characteristic curve (AUC) was used as the criterion for model accuracy. The value of AUC is normally reported between 0.5 (the model is not better than the random points) and 1 (the predicted model is very good) (SWETS 1988). The model was run for present time and then projected to the past and future periods.

\section{Results}

\section{MaxEnt distribution modeling}

Models were run in ten replicates for the five periods and the AUC values were obtained as an average value for each period as follows: LIG $(\mathrm{AUC}=0.764)$; $\mathrm{LGM}(\mathrm{AUC}=0.743)$; present $(\mathrm{AUC}=0.773)$; 2.6 for $2050(\mathrm{AUC}=0.792) ; 4.5$ for $2050(\mathrm{AUC}=0.775) ; 6.0$ for $2050(\mathrm{AUC}=0.776) ; 8.5$ for 2050 $(\mathrm{AUC}=0.764) ; 2.6$ for $2070(\mathrm{AUC}=0.783) ; 4.5$ for $2070(\mathrm{AUC}=0.787) ; 6.0$ for $2070(\mathrm{AUC}=$ 0.773 ); 8.5 for 2070 (AUC $=0.779$ ). MaxEnt predicted that the probability of these spatial patterns varied among time periods (Fig. 2). Contribution performance of each layer in the periods and models is presented in Table 1. AUC values for all models were not very good (less than 0.8 ), but were acceptable for the prediction range (SWETS, 1988). Among contributed layers, BIO11 contributed most highly for all future models and LGM. However, the greatest contributors for the present period and for LIG were $\mathrm{BIO} 8$ and $\mathrm{BIO} 4$, respectively. 
According to these results, the suitability of habitat for A. persica changed from LIG to the present. As presented in Fig. 2, the suitable area increased from LIG to LGM, even in southwestern Iran, but decreased in the present time to the central part of the Iranian Plateau. However, the projected maps show that in past periods the suitable area was restricted to western Iran and in the present time it completely changed and shifted to central Iran. Future projections in different scenarios show that southeast Iran (Baluchistan) will remain suitable for the species distribution and some parts in central Iran will be added to the present period prediction (Fig. 2).

\section{Discussion}

Our case study predictions illustrate the spatial distribution of Agamura persica in Iran at five time periods. The spatial distribution for A. persica was predicted according to MaxEnt and showed the suitable range in the central part of the Iranian Plateau. The MaxEnt algorithm is based only on presence data (VANDERWAL et al. 2009). The average predictions for all periods of time are presented in Fig. 2 (LIG, LGM, present, 2050 and 2070 respectively). The results indicate the effect of climate change on the distribution range of $A$. persica. The species was restricted to low elevation areas, where the temperature was suitable and, based on the model, temperature seasonality was the most effective factor. During the LGM suitable region increased and covered all parts of central Iran and extended into the southwest. Mean temperature of the coldest quarter influenced this prediction because the LGM was colder than LIG and these animals need moderate temperature to survive. In the present and future periods, the suitable habitat contracted, and will contract respectively, because of temperature increases causing a decrease in suitable regions. Currently, most parts of the central plateau are occupied by the species, but the models predict that it will move into the central part of Iran and into southeast Iran in

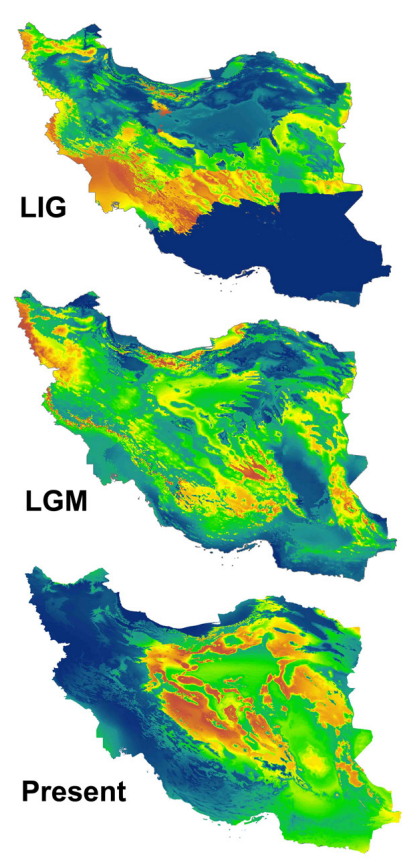

Suitability

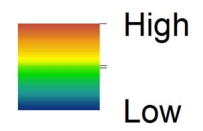





Figure 2 - Habitat suitability of Agamura persica in Iran based upon the Maxent models. All models were presented from past to future order and four future scenarios are presented in two columns. Present models were run and then projected to other times as past or future. 
TABLE 1

Contribution performance of bioclimatic layers in habitat suitability prediction from all eleven models. Bold numbers refer to the highest contributed variable on that model.

\begin{tabular}{cccccccccccc}
\hline Variables & LIG & LGM & present & $\mathbf{2 . 6} \mathbf{2 0 5 0}$ & $\mathbf{4 . 5} \mathbf{2 0 5 0}$ & $\mathbf{6 . 0} \mathbf{2 0 5 0}$ & $\mathbf{8 . 5} \mathbf{2 0 5 0}$ & $\mathbf{2 . 6} \mathbf{2 0 7 0}$ & $\mathbf{4 . 5} \mathbf{2 0 7 0}$ & $\mathbf{6 . 0} \mathbf{2 0 7 0}$ & $\mathbf{8 . 5} \_2070$ \\
\hline BIO4 & $\mathbf{5 1 , 2}$ & 4,3 & 2,2 & 32,8 & 29,3 & 33,4 & 32,8 & 38,2 & 31,7 & 33,6 & 28,7 \\
BIO6 & 5 & $\mathbf{2 8 , 9}$ & 29,8 & 14,5 & 18,4 & 13,5 & 14,2 & 12,2 & 17,6 & 15,7 & 16,5 \\
BIO8 & 9,6 & 3,6 & $\mathbf{4 8 , 9}$ & 6,4 & 4,7 & 4,6 & 5,4 & 3,2 & 5,9 & 5,6 & 5,3 \\
BIO9 & 10 & 2,1 & 0,5 & 8,8 & 4,6 & 8,5 & 5 & 7,5 & 5,1 & 4,4 & 5,7 \\
BIO11 & 24,3 & 61 & 18,6 & $\mathbf{3 7 , 5}$ & $\mathbf{4 3}$ & $\mathbf{4 0}$ & $\mathbf{4 2 , 7}$ & $\mathbf{3 8 , 9}$ & $\mathbf{3 9 , 7}$ & $\mathbf{4 0 , 7}$ & $\mathbf{4 3 , 8}$ \\
\hline
\end{tabular}

the future (2050 and 2070). The future predictions were run using four scenarios (latest IPCC report as 2.6, 4.5, 6.0, and 8.5) of $\mathrm{CO}_{2}$ level increase and resultant decrease in precipitation. These changes will tend to cause conditions to be warmer and drier than the regions that are suitable for most of the gekkonids, especially desert geckoes. MaxEnt is a useful tool for conservation purposes by predicting newly available habitats that are suitable under a changing climate; however, these predictions do not include regions not currently occupied by the species.

Finally, our results highlight the change in habitat suitability for Agamura persica in Iran from LIG to 2070, with the widest suitable areas predicted in LGM and the smallest suitable prediction is 2070 . The results indicated that mean temperature of the wettest quarter (BIO8) and mean temperature of the coldest quarter (BIO11) will have the most effect on species distribution in future. However, habitat fragmentation and dispersal may play an important role in possible effects of climate change. The distribution is affected by temperature changes related to climate change because, according to IPCC scenarios, $\mathrm{CO}_{2}$ concentration will increase until 2070 and consequently global temperatures will increase. Global warming is the challenge for conservation biology in future, but may have a positive effect in some cases (including for some lizards). Agamura persica is one of the gekkonid lizards endemic to the Iranian Plateau and found in the submountain regions (ANDERSON 1999). Northeastern Iran is predicted to be occupied by the species during present conditions, but only in a small region in the Sarakhs. This area is not predicted to change in the future and should remain as suitable (Fig. 2). Saravan, is a mountainous region and has moderate climate conditions, so it will not be influenced as much by temperature changes and therefore will be suitable for the species. However, temperature can indirectly affect the species distribution by changing the vegetation cover and richness in habitat. We investigated all habitats of the species in Iran and found that temperature will affect the suitable range for this species more in eastern Iran. Fragmentation of suitable regions will affect the species distribution in the future. If its range is fragmented by forces associated with climate change, then this could directly affect genetic differentiation for a long time.

Finally, the results of the species distribution modeling for five periods were similar but the range from LIG is slightly different from the present period and, in general, it will not change greatly for the future. We recommend that microsatellite analyses be formed among different populations of Agamura persica on the Iranian Plateau to evaluate their genetic differentiation.

\section{Acknowledgements}

We thank Ann Paterson, Muhammad Sharif Khan and Karin Tamar for reading an early draft of the manuscript and for providing valuable comments to improve the manuscript. This research was funded by a grant (no. 3/39887) from Ferdowsi University of Mashhad. We also thank the Department of Environment of Iran, which permitted us to visit all habitats of the species in Iran.

\section{References}

ANDERSON S.C. (1999). The lizards of Iran. Society for the Study of Amphibians and reptiles, Saint Louis, $442 \mathrm{pp}$. 
CAnadell J.G., Le Quéré C., Raupach M.R., Field C.B., Buitenhuis E.T., Ciais P., Conway T.J., Gillett N.P., Houghton R. \& MARLAND G. (2007). Contributions to accelerating atmospheric CO2 growth from economic activity, carbon intensity, and efficiency of natural sinks. Proceedings of the National Academy of Sciences 104: 18866-18870. https://doi.org/10.1073/pnas.0702737104

ChakRABorty S. \& NewTon A.C. (2011). Climate change, plant diseases and food security: an overview. Plant Pathology 60: 2-14. https://doi.org/10.1111/j.1365-3059.2010.02411.x

De Souza Muñoz M.e., De Giovanni R., De Siqueira M.F., Sutton T., Brewer P., Pereira R.S., CANHOS D.A.L. \& CANHOS V.P. (2011). OpenModeller: a generic approach to species' potential distribution modelling. GeoInformatica 15: 111-135. https://doi.org/10.1007/s10707-009-0090-7

Elith J., Phillips S.J., Hastie T., DudíK M., CheE Y.E. \& YATEs C.J. (2011). A statistical explanation of MaxEnt for ecologists. Diversity and Distribution 17: 43-57.

https://doi.org/10.1111/j.1472-4642.2010.00725.x

FRANKLIN J. (2010). Moving beyond static species distribution models in support of conservation biogeography. Diversity and Distribution 16: 321-330. https://doi.org/10.1111/j.1472-4642.2010.00641.x

Keil P., Belmaker J., Wilson A.M., Unitt P. \& Jetz W. (2013). Downscaling of species distribution models: a hierarchical approach. Methods in Ecology and Evolution 4: 82-94.

https://doi.org/10.1111/j.2041-210x.2012.00264.x

Kéry M., Gardner B. \& Monnerat C. (2010). Predicting species distributions from checklist data using site-occupancy models. Journal of Biogeography 37: 1851-1862.

https://doi.org/10.1111/j.1365-2699.2010.02345.x

PHILLIPS S.J., ANDERSON R.P. \& SCHAPIRE R.E. (2006). Maximum entropy modeling of species geogra-phic distributions. Ecological modeling 190: 231-259. https://doi.org/10.1016/j.ecolmodel.2005.03.026

Pounds J.A., Bustamante M.R., Coloma L.A., Consuegra J.A., Fogden M.P., Foster P.N., La Marca E., Masters K.L., Merino-Viteri A. \& Puschendorf R. (2006). Widespread amphibian extinctions from epidemic disease driven by global warming. Nature 439: 161-167. https://doi.org/10.1038/nature04246

Root T.L., Price J.T., Hall K.R., Schneider S.H., Rosenzweig C. \& PoundS J.A. (2003). Fingerprints of global warming on wild animals and plants. Nature 421: 57-60. https://doi.org/10.1038/nature01333

Sala O.E., Chapin F.S., Armesto J.J., Berlow E., Bloomfield J., Dirzo R., Huber-Sanwald E., HUENNEKE L.F., JACKSON R.B. \& KINZIG A. (2000). Global biodiversity scenarios for the year 2100. Science 287: 1770-1774. https://doi.org/10.1126/science.287.5459.1770

SWETS J.A. (1988). Measuring the accuracy of diagnostic systems. Science 240: 1285-1293.

SZCZERBAK N. \& GolubeV M. (1996). Gecko fauna of the USSR and contiguous regions. Contibutions to Herpetology 13, Society for the Study of Amphibian and Reptiles, Oxford, Ohio.

Vanderwal J., Shoo L.P., Graham C. \& Williams S.E. (2009). Selecting pseudo-absence data for presence-only distribution modeling: How far should you stray from what you know? Ecological Modeling 220: 589-594. https://doi.org/10.1016/j.ecolmodel.2008.11.010

VitT L.J. \& CALDwell J.P. (2013). Herpetology: an Introductory Biology of Amphibians and Reptiles. Academic Press.

Walther G.R., Post E., Convey P., Menzel A., Parmesan C., Beebee T.J., Fromentin J.M., HoeghGuldBERG O. \& BAIRLEIN F. (2002). Ecological responses to recent climate change. Nature 416: 389-395. https://doi.org/10.1038/416389a

Manuscript received: 1 April 2017

Manuscript accepted: 11 September 2017

Published on: 26 October 2017

Branch editor: Johan Michaux 